\title{
Esofagitis eosinofílica en el adulto: aspectos clínicos, endoscópicos, pH-métricos y manométricos
}

\author{
L. Martín Martín, C. Santander Vaquero, S. Sánchez Prudencioํㅗ J. Cantero Perona, J. P. Gisbert y \\ R. Moreno Otero
}

Servicio de Hepatogastroenterología y Centro de Investigación Biomédica en Red de Enfermedades Hepáticas y Digestivas (Ciberehd). Hospital Universitario de La Princesa. Universidad Autónoma de Madrid. 'Servicio de Aparato Digestivo. Hospital de Fuenlabrada. Madrid

\section{RESUMEN}

Objetivos: la esofagitis eosinofílica (EE) es una entidad de interés creciente caracterizada en el adulto por disfagia y episodios de impactación alimentaria recurrentes. Nuestro objetivo fue estudiar las características clínicas, endoscópicas, manométricas y pHmétricas en los pacientes con $\mathrm{EE}$, así como la relación que pudiera existir entre ellas.

Pacientes y métodos: se estudiaron de forma prospectiva durante un año los pacientes adultos diagnosticados de EE. En todos se realizó endoscopia digestiva alta con toma de biopsias, manometría esofágica estacionaria y $\mathrm{pH}$-metría ambulatoria. Se documentó además el tiempo de evolución clínica, los antecedentes de atopia, la presencia de eosinofilia periférica, la frecuencia de la disfagia y el número de desimpactaciones y complicaciones endoscópicas.

Resultados: se estudiaron 11 pacientes (edad media 35 años). Todas las endoscopias presentaron hallazgos patológicos: traquealización en 5, alteraciones mucosas en 4 , en 3 anillos esofágicos distales y en 2 estenosis esofágicas. Precisaron desimpactaciones endoscópicas 7 pacientes. Se produjeron desgarros de la mucosa esofágica durante la endoscopia en 2 pacientes. La manometría esofágica fue patológica en 6 pacientes, de estos, 5 fueron trastornos de hipomotilidad. La pH-metría fue patológica en 2 casos. Presentaron eosinofilia periférica 3 pacientes.

Conclusión: aunque los hallazgos patológicos en la endoscopia son frecuentes, no siempre justifican los episodios de impactación alimentaria característicos de la EE. Los trastornos de motilidad en forma de peristalsis esofágica ineficaz son el hallazgo manométrico más frecuente, aunque tampoco se han podido relacionar con un mayor número de episodios de disfagia o de impactaciones.

Palabras clave: Esofagitis eosinofílica. Manometría. Esofagoscopia. Impactación alimentaria.

\begin{abstract}
Objectives: eosinophilic esophagitis (EE) is a condition characterized by dysphagia and frequent food impaction in young adults. The aim of our study was to evaluate the clinical aspects, endoscopic features, pH-metric and motility disorders in EE.

Patients and methods: adult patients with EE were prospectivity included. Endoscopy with biopsy, stationary esophageal manometry, and 24-hour $\mathrm{pH}$-metry were performed. We analyzed the duration of disease, allergies, blood peripheral eosinophilia, prevalence of dysphagia, number of food impaction episodes, and complications during the endoscopic procedure.

Results: eleven male patients with a mean age of 35 years were followed. Endoscopy showed esophageal disorders in all cases: 5 esophageal felinizations, mucosal abnormalities in 4 cases, distal rings in 3 cases, and 2 esophageal stenoses. In two cases mucosal tearing during the endoscopic procedure was described. In 6 patients the manometric study showed motor disorders affecting the esophageal body, 5 of them displaying hypomotility. Two patients showed pathological gastroesophageal reflux during $\mathrm{pH}$-monitoring. Blood peripheral eosinophilia was detected in 3 patients.

Conclusion: although endoscopic abnormalities are frequently found, they do not usually explain dysphagia and food impaction episodes in EE. Ineffective esophageal peristalsis is the most prevalent manometric disorder associated with this entity, although it is not clearly related to symptom worsening either.
\end{abstract}

Key words: Eosinophilic esophagitis. Manometry. Esophagoscopy. Food impaction.

Martín Martín L, Santander Vaquero C, Sánchez Prudencio S, Cantero Perona J, Gisbert JP, Moreno Otero R. Esofagitis eosinofílica en el adulto: aspectos clínicos, endoscópicos, pH-métricos y manométricos. Rev Esp Enferm Dig 2008; 100: 476-480.

Recibido: 29-04-08.

Aceptado: 13-05-08.

Correspondencia: Leticia Martín Martín. Servicio de Aparato Digestivo. Hospital Universitario de La Princesa. C/ Diego de León, 62. 28006 Madrid.e-mail: lmleticia@yahoo.es

\section{INTRODUCCIÓN}

La esofagitis eosinofílica (EE) fue descrita por primera vez en 1977 por Landres (1) en un paciente con acalasia vigorosa, pero se ha definido como entidad propia durante la 
última década. Se caracteriza por la infiltración de la pared esofágica por leucocitos eosinófilos en ausencia de los mismos en otros tramos del tubo digestivo $(2,3) \mathrm{y}$, aunque típicamente ha sido una enfermedad pediátrica, cada vez son más numerosos los casos descritos en adultos. Es más frecuente en varones jóvenes y su forma de presentación habitual es la disfagia intermitente, a menudo acompañada de episodios de impactación alimentaria (2-4); se estima que hasta el $60 \%$ de los pacientes con EE presenta más de un episodio semanal de disfagia $(2,3)$ y que en el adulto joven la mitad de las impactaciones alimentarias pueden ser debidas a esta enfermedad (4,5). En su etiopatogenia se postula que la infiltración de la pared esofágica por leucocitos eosinófilos y su degranulación posterior en las capas musculares y nerviosas producirían las alteraciones motoras y estructurales observadas en la $\mathrm{EE}(2,6,7)$.

Todas las series estudiadas coinciden en que entre el $80-90 \%$ de los pacientes presenta hallazgos en la endoscopia, si bien la mayoría son inespecíficos $(8,9)$ y ninguno se considera patognomónico de EE (10).

Debido a que en ocasiones los episodios de impactación alimentaria no se correlacionan con los hallazgos en la esofagoscopia, el estudio de la motilidad esofágica en estos pacientes parece razonable. Los estudios de motilidad realizados hasta ahora en pacientes con EE se han recogido de forma retrospectiva en una revisión de la American Gastroenterological Association, donde se describen los resultados manométricos de 77 pacientes adultos, presentado alteraciones motoras 30 de ellos (10). Recientemente, Lucendo y cols. (3) encontraron que el 57\% de una serie de 30 pacientes adultos con EE presentaba alteraciones en la manometría de tipo hipomotilidad y el $30 \%$ un patrón de hipermotilidad. El objetivo de este trabajo fue estudiar una serie de características clínicas, hallazgos endoscópicos, manométricos y pH-métricos en los pacientes diagnosticados de EE; así como analizar si existía correlación entre ellos.

\section{MATERIAL Y MÉTODOS}

\section{Pacientes}

Se incluyeron de forma prospectiva durante 12 meses los pacientes diagnosticados de EE mediante criterios histológicos, en el contexto clínico adecuado y excluyendo otras causas de infiltración eosinófila esofágica; es decir, siguiendo así las recomendaciones de la American Gastroenterological Association. Se consideró diagnóstico de EE la presencia de 15 o más leucocitos eosinófilos en al menos uno de los campos de gran aumento (x400) de las biopsias esofágicas obtenidas por endoscopia. La sospecha clínica se basó en episodios de impactación alimentaria o en antecedentes de disfagia recurrente en adultos jóvenes. Todos los pacientes fueron informados, aceptaron y firmaron el consentimiento informado.

Se realizó un cuestionario a cada paciente en el que se recogió la edad, los antecedentes personales y familiares de primer grado de atopia, el momento del inicio de los síntomas, la frecuencia mensual de la disfagia y el número de impactaciones alimentarias, en caso de presentarlas, que habían requerido desimpactaciones endoscópicas. Se documentó la presencia de eosinofilia en sangre periférica, considerándose anormales las cifras superiores al 7\% del total del recuento leucocitario.

Se realizó manometría esofágica estacionaria y $\mathrm{pH}-$ metría ambulatoria en todos los pacientes. Para ello se indicó la suspensión de todos los fármacos que afectan a la motilidad esofágica con una antelación de al menos 72 horas, y los inhibidores de la bomba de protones se suspendieron al menos con una semana de antelación a la realización del estudio.

\section{Estudio manométrico}

Se realizó manometría esofágica estacionaria mediante un sistema de perfusión hidroneumocapilar de baja distensibilidad, usando una sonda multicatéter de polivinilo de ocho canales (diámetro interno de $0,8 \mathrm{~mm}$ y externo de 3,2 $\mathrm{mm}$ ) con ocho orificios orientados radialmente separados $2 \mathrm{~cm}$ entre sí. Cada canal se conectó con un transductor externo y mediante el sistema de infusión se perfundió agua destilada a una velocidad constante de 0,6 ml/min (Sistema Solar GI Manometry de Medical Measurament SystemsMMS). Se realizó la manometría según la técnica de retirada del catéter a intervalos de $0,5 \mathrm{~cm}$ como describe Castell (11). La interpretación de los resultados se hizo siguiendo los criterios usados por Leite, Ho y Tutuian (12-14).

\section{pH-metría}

Para realizar la pH-metría esofágica de 24 horas se utilizó un registrador Holter ambulatorio Mark III (Synectics Medical, Irving, Tx), con una frecuencia de registro cada 4 segundos, conectado a un electrodo de antimonio monocristalino y software específico (Polygraf version 5.0. Synectics Medical, Estocolmo, Suecia). El electrodo de antimonio fue colocado $5 \mathrm{~cm}$ por encima de la parte superior del esfínter esofágico inferior (EEI), previamente localizada por manometría esofágica, y después de la calibración de la sonda a pH 1,07 y 7,01. Consideramos la prueba patológica cuando el porcentaje de exposición ácida total en tercio distal superaba el $4 \%$ y/o el score de De Meester estaba por encima de 14,24 puntos $(15,16)$.

\section{RESULTADOS}

\section{Variables clínicas}

Se diagnosticaron 11 pacientes con EE. Todos fueron varones con una edad media de $35 \pm 3$ años. Seis de ellos (54\%) no referían antecedentes personales de atopia. Tres 
pacientes eran alérgicos al polen, dos a gramíneas, tres eran alérgicos a los ácaros (uno de ellos refería además alergia al kiwi y a la cerveza) y otros dos referían antecedentes de asma. Cuatro (36\%) pacientes referían antecedentes familiares de primer grado de atopia. Tres $(27 \%)$ pacientes presentaron eosinofilia en sangre periférica, aunque leve, con cifras de $0,5 \times 10^{3} /$ microlitro, $0,7 \times$ $10^{3} /$ microlitro y $1,0 \times 10^{3} /$ microlitro (valores normales inferiores a $0,5 \times 10^{3} /$ microlitro).

El tiempo transcurrido desde el inicio de los síntomas varió entre 1 y 20 años; un paciente refirió concretamente el inicio de los síntomas tras presentar una infección de vías respiratorias bajas y otro de los pacientes presentaba disfagia únicamente con la ingesta de yema de huevo y legumbres. El número de episodios de disfagia mensuales variaba entre 1 y 12 . De los 11 pacientes diagnosticados, $7(64 \%)$ requirieron desimpactaciones alimentarias endoscópicas urgentes en al menos una ocasión.

\section{Hallazgos endoscópicos}

El 100\% de las endoscopias mostraron alteraciones. El hallazgo más frecuente fue el esófago traquealizado en $5(45 \%)$ de los casos. Se describieron alteraciones mucosas en forma de exudados blanquecinos en 4 (36\%) pacientes, en $3(27 \%)$ se encontraron anillos de Schatzky y en $2(27 \%)$ casos estenosis de la luz esofágica, una a nivel del esófago medio y otra en el esófago distal; pero ninguna de estas impedía el paso del endoscopio. En 2 (27\%) casos se produjeron desgarros de la mucosa esofágica durante el procedimiento, uno de ellos a nivel de una estenosis y el otro en la unión esófago-gástrica, aunque en ambos casos el sangrado fue de escasa cuantía y autolimitado.

\section{Estudio manométrico y pH-métrico}

La manometría mostró en el 100\% de los casos un esfínter esofágico superior con presiones normales y un coeficiente de sinergia faringoesfinteriano normal. El estudio del esfínter esofágico inferior fue normal en 9 $(81 \%)$ de los casos, los otros $2(18 \%)$ eran hipotensos (grado leve), aunque con un comportamiento dinámico adecuado y sin que esto se relacionase con la presencia de reflujo gastroesofágico patológico en la pH-metría. En el estudio del cuerpo esofágico se diagnosticaron alteraciones de la motilidad en $6(54 \%)$ de los 11 pacientes; de estos, 5 (83\%) fueron trastornos de hipomotilidad en forma de peristalsis esofágica ineficaz y un paciente presentó hipercontractilidad del cuerpo esofágico en forma de espasmo esofágico difuso. El diagnóstico de peristalsis esofágica ineficaz se basó en la presencia de al menos un $30 \%$ de degluciones húmedas seguidas de ondas de baja amplitud (inferior a $30 \mathrm{mmHg}$ ) o no transmitidas en esófago distal $(12,13)$. El diagnóstico de espasmo esofágico difuso se basó en la presencia de contracciones simultáneas en el esófago distal en al menos un $20 \%$ de las degluciones húmedas (14).

La pH-metría esofágica ambulatoria de un canal demostró reflujo ácido patológico en 2 (18\%) de los casos, ambos en bipedestación. Los hallazgos descritos se resumen en la tabla I.

\section{CONCLUSIÓN}

La EE es una enfermedad de interés creciente que afecta predominantemente a adultos jóvenes de sexo masculino y cuya prevalencia real es desconocida $(1,2,4,6)$. En su patogenia parece existir una respuesta aberrante a alérgenos $(1,2,4,8,9)$, que explica que el $50 \%$ de los pacientes con EE en edad pediátrica presenten síntomas de atopia. En adultos esta asociación varía entre un 29-60\% y también se encuentra entre los familiares de primer grado (17-20), aunque se desconoce en qué proporción. La EE sugiere que, aunque clásicamente se pensaba que el esófago se comportaba como un órgano sin actividad inmunocompetente, el infiltrado eosinofílico puede ser capaz de desarrollar una función inmunológica específica con repercusiones patológicas $(2,20)$; de ahí los beneficios del estudio alergológico en estos pacientes, tal y como indican Armisén y cols. (21). En nuestra serie todos los pacientes diagnosticados de EE fueron de sexo masculino, con edades comprendidas entre 22 y 43 años. El $45 \%$ de los pacientes presentaba antecedentes personales de atopia y el 36\% refería antecedentes en familiares de primer grado. La eosinofilia periférica está presente en el $10-50 \%$ de los adultos con EE $(2,7)$, pero actualmente no se considera un criterio diagnóstico ni se ha demostrado que esté relacionada con la evolución de la enfermedad (10). De los once pacientes, tres presentaron eosinofilia en sangre periférica, aunque leve.

Además de la etiología alérgica, algunos autores han estudiado la relación que pudiera existir entre la enfermedad por reflujo gastroesofágico y la EE, fenómeno patogénico que está aún por dilucidar $(6,22)$. En la bibliografía internacional se recogen 91 adultos diagnosticados de $\mathrm{EE}$ a los que se les realizó pH-metría esofágica, de ellos, aproximadamente el $18 \%$ de los casos tenía reflujo gastroesofágico ácido patológico (10). En nuestra serie, 2 pacientes $(18,1 \%)$ presentaron reflujo ácido patológico en la pH-metría, en concordancia por tanto con lo descrito hasta ahora por otros autores $(10,23)$.

En el estudio manométrico se documentaron dos casos de hipotensión del esfínter esofágico inferior, pero ninguno de ellos asoció reflujo gastroesofágico patológico en la pH-metría de 24 horas. El esfínter esofágico superior fue normal en todos los casos. En el estudio del cuerpo esofágico encontramos que el $54,5 \%$ de los pacientes de la serie presentó trastornos motores, cifra algo superior a las descritas hasta ahora por otros autores (10). Todos los trastornos que hallamos, excepto en un caso, fueron de tipo hipo- 
Tabla I. Hallazgos clínicos, endoscópicos, manométricos y pH-métricos en pacientes con esofagitis eosinofílica

\begin{tabular}{|c|c|c|c|c|c|c|c|c|c|c|c|}
\hline & $\begin{array}{l}\text { Edad } \\
\text { (años) }\end{array}$ & $\begin{array}{l}\text { Antecedentes } \\
\text { personales de } \\
\text { atopia }\end{array}$ & $\begin{array}{l}\text { Antecedentes } \\
\text { familiares de } \\
\text { atopia }\end{array}$ & $\begin{array}{l}\text { Años de } \\
\text { evolución clínica }\end{array}$ & $\begin{array}{l}\text { Episodios mensuales } \\
\text { de disfagia }\end{array}$ & $\begin{array}{c}\text { Desimpactaciones } \\
\text { endoscópicas }\end{array}$ & $\begin{array}{l}\text { Hallazgos } \\
\text { endoscópicos }\end{array}$ & EES & $\begin{array}{c}\text { Manometría } \\
C\end{array}$ & EEl & pH-metría \\
\hline Caso 1 & 42 & No & No & 2 & 4 & 3 & $\begin{array}{l}\text { Exudados blanquecinos } \\
\text { Hernia hiatal }\end{array}$ & Normal & EED & Normal & NRGE \\
\hline Caso 2 & 30 & $\begin{array}{l}\text { Asma } \\
\text { Gramíneas } \\
\text { Polen }\end{array}$ & No & 3 & 1 & 1 & $\begin{array}{l}\text { Traquealización } \\
\text { Estenosis media }\end{array}$ & Normal & PEl & Normal & RGE \\
\hline Caso 3 & 29 & $\begin{array}{l}\text { Polen } \\
\text { Cerveza } \\
\text { Kiwi }\end{array}$ & No & 3 & 1 & 2 & $\begin{array}{l}\text { Traquealización } \\
\text { Anillo distal }\end{array}$ & Normal & Normal & Normal & NRGE \\
\hline Caso 4 & 25 & $\begin{array}{l}\text { Polen } \\
\text { Ácaros }\end{array}$ & Sí & 1 & 12 & 0 & $\begin{array}{l}\text { Traquealización } \\
\text { Anillo distal }\end{array}$ & Normal & Normal & Normal & NRGE \\
\hline Caso 5 & 22 & No & No & 15 & 0 & 1 & $\begin{array}{l}\text { Exudados blanquecinos } \\
\text { Hernia hiatal }\end{array}$ & Normal & PEl & Hipotenso & NRGE \\
\hline Caso 6 & 34 & No & No & 7 & 5 & 2 & $\begin{array}{c}\text { Estenosis distal } \\
\text { Esofagitis distal } \\
\text { con lesión ulcerada } \\
\text { Anillo distal }\end{array}$ & Normal & PEl & Hipotenso & NRGE \\
\hline Caso 7 & 31 & No & No & 1 & Persistente & 0 & Exudados blanquecinos & Normal & Normal & Normal & NRGE \\
\hline Caso 8 & 52 & Asma & Sí & 1 & 4 & 0 & Exudados blanquecinos & Normal & Normal & Normal & NRGE \\
\hline Caso 9 & 53 & $\begin{array}{l}\text { Ácaros } \\
\text { Gramíneas }\end{array}$ & Sí & 4 & 1 cada 6 meses & 0 & Esofagitis distal grado A & Normal & PEl & Normal & NRGE \\
\hline Caso 10 & 24 & No & No & 4 & 1 & 1 & Traquealización & Normal & Normal & Normal & RGE \\
\hline Caso 11 & 44 & No & Sí & 10 & 12 & 2 & Traquealización & Normal & PEI & Normal & NRGE \\
\hline
\end{tabular}

EES: esfínter esofágico superior; EEl: esfínter esofágico inferior; C: cuerpo esofágico; PEl: peristalsis esofágica ineficaz; EED: espasmo esofágico difuso; RGE: reflujo gastroesofágico patológico; NRGE: ausencia de reflujo gastroesofágico.

motilidad y cumplían criterios de peristalsis esofágica ineficaz. Incluso en población con sintomatología de reflujo gastroesofágico, la prevalencia de la peristalsis esofágica ineficaz se sitúa alrededor del 30\% (24), cifra inferior a la que hallamos en nuestra serie de pacientes con EE.

Respecto a los hallazgos endoscópicos, se han descrito el esófago traquealizado, los anillos distales, las estenosis, las bandas longitudinales, los exudados mucosos o la fragilidad de la mucosa como las alteraciones más frecuentemente encontradas, sin que ninguna de ellas sea patognomónica de la EE $(10,11)$. La mayoría de estos hallazgos también estaba presente en nuestro estudio. Aunque algunos autores refieren una mayor incidencia de complicaciones durante la endoscopia (7), no existen cifras al respecto; en nuestra serie se produjeron dos desgarros mucosos, uno de ellos a nivel de una estenosis media y otro en la unión esofagogástrica. Sin embargo, no siempre se encuentran alteraciones endoscópicas esofágicas que justifiquen los episodios de impactación alimentaria; es más, Prasad y cols. (25) refieren que la endoscopia es normal en el $10 \%$ de los pacientes con EE que presentan impactaciones alimentarias.

La forma de presentación principal de la EE en el adulto es la disfagia, acompañada frecuentemente de episodios de impactaciones alimentarias esofágicas. En nuestra serie, el número de episodios de disfagia varió entre ninguno y doce al mes, sin que los pacientes con mayor número de episodios refiriesen un mayor tiempo de evolución clínica ni tampoco una mayor frecuencia de impactaciones endoscópicas. Por otro lado, el paciente que más desimpactaciones endoscópicas había requerido refería una evolución sólo de dos años, en la endoscopia no se encontraron alteraciones estructurales que justificasen la impactación, mientras que la manometría esofágica reveló un trastorno de hipermotilidad de tipo espasmo esofágico difuso. De los tres pacientes con menor tiempo de evolución clínica (un año), dos presentaban sólo hallazgos mucosos en la endoscopia y el tercero traquealización; ninguno de los tres había requerido desimpactaciones endoscópicas ni presentaron alteraciones en la manometría.

Finalmente, aunque las alteraciones endoscópicas en los pacientes con EE son casi una constante, no siempre justifican la disfagia y los episodios de impactación alimentaria. El trastorno motor más frecuente hallado en nuestro trabajo es la peristalsis esofágica ineficaz, aunque tampoco se ha podido relacionar con un mayor número de episodios de disfagia o impactaciones. Es difícil, dado el escaso número de pacientes diagnosticados de EE, establecer una secuencia en la evolución de esta enfermedad; se desconoce si las alteraciones estructurales preceden a las motoras o, por el contrario, la infiltración de los leucocitos eosinófilos en la pared esofágica induce alteraciones en la motilidad que, de forma mantenida, darían lugar a los cambios estructurales. 


\section{AGRADECIMIENTOS}

\author{
Ciberhed está financiado por el Instituto de Salud Car- \\ los III.
}

\section{BIBLIOGRAFÍA}

1. Landres RT, Kuster GG, Strum WB. Eosinophilic esophagitis in a patient with vigorous achalasia. Gastroenterol 1978; 74 (6): 1298-301.

2. Furuta GT, Straumann A. The pathogenesis and management of eosinophilic oesophagitis. Aliment Pharmacol Ther 2006; 24: 173-82.

3. Lucendo AJ, Pascual-Turrión JM, Navarro M, Comas C, Castillo P, Letrán A, et al. Endoscopic, bioptic, and manometric findings in eosinophilic esophagitis before and after steroid therapy: a case series. Endoscopy 2007; 39 (9): 765-71.

4. Kerlin P, Jones D, Remedios M, Campbell C. Prevalence of eosinophilic esophagitis in adults with food bolus obstruction of the oesophagus. J Clin Gastroenterol 2007; 41 (4): 356-61.

5. Potter JW, Saeian K, Staff D, et al. Eosinophilic esophagitis in adults: an emerging problem with unique oesophageal features. Gastrointest Endosc 2004; 59 (3): 355-61.

6. Spechler SJ, Genta RM, Souza RF. Thoughts on the complex relationship between gastroesophageal reflux disease and eosinophilic esophagitis. Am J Gastroenterol 2007; 102: 1301-6.

7. Samuel Nurko MD, Glenn T, Furuta MD. Eosinophilic esophagitis. GI Motility online 2006. Disponible en: http://www.nature.com/ gimo/contents/pt1/full/gimo49.html.

8. Straumann A, Spichtin H, Bucher KA, Herr P, Simon H. Eosinophilic esophagitis: red on microscopy, white on endoscopy. Digestion 2004; 70: 109-16.

9. Yan BM, Schaffer EA. Eosinophilic esophagitis: a newly established cause of dysphagia. World J Gastroenterol 2006; 12 (15): 2328-34.

10. Furuta GT, Liacouras CA, Collins MH, Gupta SK, Justinich C, Putnam PE, et al. First International Gastrointestinal Eosinophil Research Symposium (FIGERS) Subcommittees. Eosinophilic esophagitis in children and adults: a systematic review and consensus recommendations for diagnosis and treatment. AGA Institute. Gastroenterol 2007; 133: 1342-63.

11. Castell JA, Dalton CB. Esophageal manometry. In: Castell DO, edi- tor. The Esophagus. Boston: Little Brown and Company; 1992. p. 143-60

12. Ho SC, Chang CS, et al. Ineffective esophageal motility disorder in gastroesophageal reflux disease. Dig Dis Sci 2002; 47: 652-6.

13. Leite LP, Johnston BT, Barret J, Castell JA, Castell DO. Ineffective oesophageal motility. The primary finding in patients with nonespecific oesophageal motility disorder. Dig Dis Sci 1997; 42: 1859-65.

14. Tutuian R, Castell DO. Esophageal spam-diagnosis and management. Aliment Pharmacol Ther 2006; 23 (10): 1393-402.

15. Marnie I, Tutuian R, Castell DO. Comparison between the combined analysis and the Demeester score to predict response to PPI therapy. J Clin Gastroenterol 2006; 40 (7): 602-5.

16. Hirano I, Ritcher JE and the Practice Parameters Committee of American College of Gastroenterology. ACG Practice Guidelines: esophageal reflux testing. Am J Gastroenterol 207; 102: 668-85.

17. Orestein SR, Shalaby TM, Di Lorenzo C, Putnam PE, Sigurdsson L, Kocohis S. The spectrum of eosinophilic esophagitis beyond infancy: a clinical series of 30 children. Am J Gastrointest 2000; 95: 1422-30.

18. Liacouras C, Ruchelli E. Eosinophilic esophagitis. Curr Opin Pediatr 2004; 16: 560-5.

19. Liacouras CA, Spergel JM, Ruchelli E, et al. Eosinophilic esophagitis: a 10-year experience in 381 children. Clin Gastroenterol Hepatol 2005; 3: 1198-206.

20. Rothenber ME. Pathogenesis and clinical features of eosinophilic esophagitis. J Allergy Clin Immunol 2001; 108: 891-4.

21. Armisén M, Vidal C, López-Rosés L, Rodríguez V, Bartolomé B. Eosinophilic esophagitis due to allergy to sheep and gota milk proteins. Rev Esp Enferm Dig 2008; 100: 53-6.

22. Ronkainen J, Talley NJ, Aro P, Storsdrubb T, Johansson SE, Lind T, et al. Prevalence of esophageal eosinophilia and eosinophilic esophagitis in adults: Kalisanda's study in populations. Gut 2007; 1 (4): 219-23.

23. Lucendo Villarín AJ, Carrión Alonso G, Navarro Sánchez M, Martín Chavarri, Gómez Senent S, Castillo Grau P, et al. Eosinophilic esophagitis in adults, an emerging cause of dysphagia. Description of 9 cases. Rev Esp Enferm Dig 2005; 97: 229-39.

24. Kahrilas PJ, Pandolfino JF. Ineffective oesophageal motility does not equate to GERD. Am J Gastroenterol 2003; 98 (4): 771-6.

25. Prasad SF, Di Biaise JK, Kim HJ, De Petris G, Crowell MD, Fleischer DE, et al. Patient characteristics, clinical, endoscopic, and histologic findings in adult eosinophilic esophagitis: a case series and systematic review of the medical literature. Dis Esophagus 2007; 20 (4): $311-9$. 